\title{
A Novel Method for Analytical Solutions of Fractional Partial Differential Equations
}

\author{
Mehmet Ali Akinlar ${ }^{1}$ and Muhammet Kurulay ${ }^{2}$ \\ ${ }^{1}$ Department of Mathematics, Bilecik Seyh Edebali University, 11210 Bilecik, Turkey \\ ${ }^{2}$ Department of Mathematics, Yildiz Technical University, 34220 Istanbul, Turkey \\ Correspondence should be addressed to Muhammet Kurulay; mkurulay@yildiz.edu.tr
}

Received 13 June 2013; Accepted 25 June 2013

Academic Editor: Chaudry Masood Khalique

Copyright (c) 2013 M. A. Akinlar and M. Kurulay. This is an open access article distributed under the Creative Commons Attribution License, which permits unrestricted use, distribution, and reproduction in any medium, provided the original work is properly cited.

\begin{abstract}
A new solution technique for analytical solutions of fractional partial differential equations (FPDEs) is presented. The solutions are expressed as a finite sum of a vector type functional. By employing MAPLE software, it is shown that the solutions might be extended to an arbitrary degree which makes the present method not only different from the others in the literature but also quite efficient. The method is applied to special Bagley-Torvik and Diethelm fractional differential equations as well as a more general fractional differential equation.
\end{abstract}

\section{Introduction}

Fractional calculus is a significantly important and useful branch of mathematics having a broad range of applications at almost any branch of science. Techniques of fractional calculus have been employed at the modeling of many different phenomena in engineering, physics, and mathematics. Problems in fractional calculus are not only important but also quite challenging which usually involves hard mathematical solution techniques (see, e.g., [1]). Unfortunately, a general solution theory for almost each problem in this area has yet to be established. Each application venue has developed its own approaches and implementations. As a consequence, a single standard method for the problems in fractional calculus has not emerged. Therefore, finding reliable and efficient solution techniques along with fast implementation methods is a significantly important and active research area.

In the literature, a number of methods have been developed for the numerical or analytical solutions for FPDEs. We can list some of these methods as follows: Adomian decomposition method [2], the collocation method [3], the fractional differential transform method [4], homotopy analysis method [5], homotopy perturbation method [6], and some other methods $[7,8]$ listed on the references of these papers. In this paper, we present a new method for the analytical solutions of FPDEs. The solutions are expressed as a finite sum of a vector type functional. By employing MAPLE software, it is shown that the solutions might be extended to an arbitrary degree which makes the present method not only different from the others in the literature but also quite efficient. The method is applied to special Bagley-Torvik and Diethelm fractional differential equations as well as a more general fractional differential equation.

Now let us briefly review some significant concepts in fractional calculus. The fractional calculus is a name for the theory of integrals and derivatives of arbitrary order, which unifies and generalizes the notions of integer-order differentiation and $n$-fold integration. We have well-known definitions of a fractional derivative of order $\alpha>0$ such as Riemann-Liouville, Grunwald-Letnikow, Caputo, and generalized functions approach [9]. The most commonly used definitions of fractional derivative belongs to RiemannLiouville and Caputo. For the purpose of this paper, Caputo's definition of fractional differentiation will be used, taking the advantage of Caputo's approach that the initial conditions for fractional differential equations with Caputo's derivatives take on the traditional form as for integer-order differential equations. We give some basic definitions and properties of the fractional calculus theory which were used through paper. 
Definition 1. A real function $f(x), x>0$, is said to be in the space $C_{\mu}, \mu \in R$ if there exists a real number $(p>\mu)$, such that $f(x)=x^{p} f_{1}(x)$, where $f_{1}(x) \in C[0, \infty)$, and it is said to be in the space $C_{\mu}^{m}$ if $f^{m} \in C_{\mu}, m \in N$.

Definition 2. The Riemann-Liouville fractional integral operator of order $\alpha \geq 0$ of a function $f \in C_{\mu}, \mu \geq-1$ is defined as

$$
\begin{gathered}
J_{0}^{v} f(x)=\frac{1}{\Gamma(v)} \int_{0}^{x}(x-t)^{v-1} f(t) d t, \quad v>0, \\
J^{0} f(x)=f(x) .
\end{gathered}
$$

It has the following properties.

For $f \in C_{\mu}, \mu \geq-1, \alpha, \beta \geq 0$, and $\gamma>1$,

(1) $J^{\alpha} J^{\beta} f(x)=J^{\alpha+\beta} f(x)$,

(2) $J^{\alpha} J^{\beta} f(x)=J^{\beta} J^{\alpha} f(x)$,

(3) $J^{\alpha} x^{\gamma}=(\Gamma(\gamma+1) / \Gamma(\alpha+\gamma+1)) x^{\alpha+\gamma}$.

The Riemann-Liouville fractional derivative is mostly used by mathematicians but this approach is not suitable for the physical problems of the real world since it requires the definition of fractional order initial conditions, which have no physically meaningful explanation yet. Caputo introduced an alternative definition, which has the advantage of defining integer order initial conditions for fractional order differential equations.

Definition 3. The fractional derivative of $f(x)$ in the Caputo sense is defined as

$$
\begin{aligned}
D_{*}^{v} f(x) & =J_{a}^{m-v} D^{m} f(x) \\
& =\frac{1}{\Gamma(m-v)} \int_{0}^{x}(x-t)^{m-v-1} f^{(m)}(t) d t,
\end{aligned}
$$

for $m-1<v<m, m \in N, x>0, f \in C_{-1}^{m}$.

Lemma 4. If $m-1<\alpha<m, m \in N$, and $f \in C_{\mu}^{m}, \mu \geq-1$, then

$$
\begin{gathered}
D_{*}^{\alpha} J^{\alpha} f(x)=f(x), \\
J^{\alpha} D_{*}^{v} f(x)=f(x)-\sum_{k=0}^{m-1} f^{k}\left(0^{+}\right) \frac{x^{k}}{k !}, \quad x>0 .
\end{gathered}
$$

The Caputo fractional derivative is considered here because it allows traditional initial and boundary conditions to be included in the formulation of the problem.

Definition 5. For $m$ to be the smallest integer that exceeds $\alpha$, the Caputo time-fractional derivative operator of order $\alpha>0$ is defined as

$$
D_{* t}^{\alpha} u(x, t)=\frac{\partial^{\alpha} u(x, t)}{\partial t^{\alpha}}
$$

$$
=\left\{\begin{array}{lr}
\frac{1}{\Gamma(m-\alpha)} \int_{0}^{t}(t-\xi)^{m-\alpha-1} \frac{\partial^{m} u(x, \xi)}{\partial \xi^{m}} d \xi, \\
\frac{\partial^{m} u(x, t)}{\partial t^{m}}, & \text { for } m-1<\alpha<m,
\end{array}\right.
$$

and the space-fractional derivative operator of order $\beta>0$ is defined as

$$
\begin{aligned}
D_{* x}^{\alpha} u(x, t) & =\frac{\partial^{\beta} u(x, t)}{\partial x^{\beta}} \\
& =\left\{\begin{array}{lr}
\frac{1}{\Gamma(m-\beta)} \int_{0}^{x}(x-\theta)^{m-\beta-1} \frac{\partial^{m} u(\theta, t)}{\partial \theta^{m}} d \theta, \\
\frac{\partial^{m} u(x, t)}{\partial x^{m}}, & \text { for } m-1<\beta<m,
\end{array}\right.
\end{aligned}
$$

Another concept which plays a very significant role in the fractional calculus is the Gamma function. Next we briefly overview the definition and some important properties of Gamma function.

Definition 6. For $0<n<\infty$, the integral (sometimes known as Euler integral) defined as

$$
\Gamma(n)=\int_{0}^{\infty}(-\log (t))^{(n-1)} d t
$$

is said to be Gamma function. From (6), it is clear that for $n>0$,

$$
\Gamma(n)=\int_{0}^{\infty} t^{(n-1)} e^{-t} d t
$$

Again for $n>0$, the function defined as

$$
\Gamma(n)=2 \int_{0}^{\infty} t^{(2 n-1)} e^{-t^{2}} d t
$$

is known as the Gamma function. Detailed information about the significant features of the Gamma function might be obtained from any well-written differential equation book.

Organization of the paper is in the following way. Firstly, we overview basic concepts of fractional derivative. Because we employ Caputo sense derivative, we describe it in detail. Secondly, we introduce a new method for analytical solutions of FPDEs. In the third section, we illustrate three computational examples as the application of the present method and complete the paper with a discussion section.

\section{A Novel Method for Analytical Solutions of FPDEs}

Let us consider the FPDE given as

$$
\begin{array}{r}
D^{n} x(t)=f\left(t, x(t), D^{\alpha_{1}} x(t), D^{\alpha_{2}} x(t), \ldots, D^{\alpha_{m}} x(t)\right), \\
t>0,
\end{array}
$$


where $\alpha_{i}$ is a real number for every $i$ and $n$ is a natural number. Initial conditions for this equation are given by

$$
\begin{array}{r}
D^{j} x(0)=a_{j}, \quad j=0,1, \ldots, n-1, \\
0<\alpha_{1}<\alpha_{2}<\cdots<\alpha_{m}<n .
\end{array}
$$

Writing

$$
y_{i}(t)=D^{\alpha_{i}} x(t)
$$

we can express (9) as

$$
D^{n} x(t)=f\left(t, y_{0}(t), y_{1}(t), \ldots, y_{m}(t)\right), \quad t>0 .
$$

Now let us assume that the solution of (12) is given by

$$
y=y_{0}+y_{1} x^{\alpha_{1}}+y_{2} x^{\alpha_{2}}+\cdots+e x^{\alpha_{n}}
$$

where $y$ is an $m$ dimensional vector function and $e$ is also a vector type function having the same dimension with $y$. If the solution (13) is plugged into (12) and the higher order derivatives are ignored, a linear equation expressed in terms of $e$ is obtained. As a consequence, $e$ is plugged in (13) and solution is obtained. If the same process is repeated for the higher order derivatives, series solution of (9) (or (13)) from an arbitrary degree is obtained.

Now in order to express the solution in a new power series form, let us explain the application of this method to the power series. When (13) is plugged into (9), we can write

$$
\begin{aligned}
f(x)= & f_{0}+f_{1} x^{\alpha_{1}}+f_{2} x^{\alpha_{2}}+\cdots \\
& +\left(f_{n}+p_{1} e_{1}+p_{2} e_{2}+\cdots+p_{m} e_{m}\right) x^{\alpha_{n}},
\end{aligned}
$$

where $p_{1}, p_{2}, \ldots, p_{m}$ are constant real numbers, $e_{1}, e_{2}, \ldots, e_{m}$ are the bases of $e$, and $m$ is the dimension of the vector $e$. It is clear that using (13) it is possible to define that

$$
y_{i}=y_{i, 0}+y_{i, 1} x^{\alpha_{1}}+y_{i, 2} x^{\alpha_{2}}+\cdots+e_{i} x^{\alpha_{n}} .
$$

If $y$ and its derivatives are written at (14), one obtains

$$
f_{i}(x)=f_{i, n}+p_{i, 1} e_{1}+\cdots+\left(p_{i, m} e_{m}\right) x^{\alpha_{n}-j}+O\left(x^{\alpha_{n}-j+1}\right),
$$

where $f_{i}$ is an element of $f\left(y, y^{\prime}, x\right)$ appearing at (9) and $j$ is an index which is only 1 less than the degree of the highest derivative given in the initial problem. By solving (16), one obtains $e_{i}$, for each $i=1,2, \ldots, m$. By writing these $e_{i}$ values at (15), for each $i$, the polynomial $y_{i}$ having the degree of $n$ is obtained. This gives us power series solution of (9) from an arbitrary order. Finally let us suppose that step size of $x$ is $h$. If this value is plugged in $y$ and $y^{\prime}, y$ and $y^{\prime}$ are obtained at the point of $x=x_{0}+h$. Repeating the same process, one obtains solution of (9).

In the next section, we illustrate the application of this new and novel method to the analytical solutions of some FPDEs.

\section{Computational Applications}

Example 7. In the first example, we consider a special case of Bagley-Torvik equation

$$
\frac{d^{2} y}{d x^{2}}+\frac{d^{3 / 2} y}{d x^{3 / 2}}+y=1+x
$$

with the initial conditions

$$
y(0)=1, \quad y^{\prime}(0)=1 .
$$

Now bearing in mind the aforementioned solution procedure, let us assume that the solution of (17) is given by

$$
y=y_{0}+y_{1} x^{1 / \alpha}+y_{2} x^{2 / \alpha}+y_{3} x^{3 / \alpha}+y_{4} x^{4 / \alpha} .
$$

For $\alpha=2$, from (13) we can write that

$$
y=1+x+e x^{2}
$$

Taking the derivatives of $y$, we get

$$
\frac{\Gamma(3)}{\Gamma(1.5)} e x^{0.5}+1+x+e x^{2}=1+x
$$

which implies that $e=0$. In a similar way, one obtains

$$
y=1+x+e x^{5 / \alpha}
$$

Again by taking the derivatives of $y$, one obtains that $e=0$. In the next step, we have

$$
y=1+x+y_{6} x^{6 / \alpha}
$$

which implies that $e=0$. Repeating this procedure, one obtains the solution of (17) as

$$
y=1+x
$$

Example 8. In this example, we consider the initial value problem studied by Diethelm and given as

$$
D^{0.5} y(x)=y(x)+x^{2}+\frac{2}{\Gamma(2.5)} x^{1.5}
$$

with initial conditions

$$
y(0)=0 .
$$

Let us suppose that the solution of (25) is given by $y=e x$. When we write this solution in the original equation (25), we get $e=0$. In the same way, if we consider the solution

$$
y=e x^{2},
$$

we obtain $e=1$. Again by considering the solution

$$
y=x^{2}+e x^{3}
$$

we obtain $e=0$. Repeating the procedure in this way, we obtain the solution of the initial value problem (25) as

$$
y=x^{2}
$$

which is also exact solution of the problem.

In the last example, we consider a more general example to illustrate the application of the novel method. 
Example 9. Let us consider the equation

$$
\begin{aligned}
& a(t) D^{2} x(t)+b(t) D^{\alpha_{2}} x(t) \\
& \quad+c(t) D x(t)+e(t) D^{\alpha_{1}} x(t)+k(t) x(t) \\
& =f(t), \quad 0<\alpha_{1}<\alpha_{2}<1,
\end{aligned}
$$

where

$$
\begin{aligned}
f(t)= & -a-\frac{b(t)}{\Gamma\left(3-\alpha_{2}\right)} t^{2-\alpha_{2}}-c(t) t \\
& -\frac{e(t)}{\Gamma\left(3-\alpha_{1}\right)} t^{2-\alpha_{1}}+k(t)\left(2-\frac{1}{2} t^{2}\right),
\end{aligned}
$$

with the initial conditions

$$
x(0)=2, \quad x^{\prime}(0)=0 .
$$

Following the steps of aforementioned solution algorithm, one can obtain the solution of (30) as

$$
x(t)=2-\frac{1}{2} t^{2}
$$

which is also the exact solution of this problem.

\section{Conclusion}

A new technique for the analytical solutions of FPDEs has been successfully developed in this paper. By employing MAPLE software, it is shown that the solutions might be extended to an arbitrary degree which makes the present method not only different from the others in the literature but also quite efficient. The method is applied to special BagleyTorvik and Diethelm fractional partial differential equations as well as a more general fractional differential equation. Experimental results prove that the present method is a useful and highly efficient technique.

\section{Conflict of Interests}

The authors declare no conflict of interests.

\section{References}

[1] J. Sabatier, C. Ionescu, J. K. Tar, and J. A. T. Machado, "New challenges in fractional systems," Mathematical Problems in Engineering, vol. 2013, Article ID 239378, 2 pages, 2013.

[2] S. Momani and R. Qaralleh, "An efficient method for solving systems of fractional integro-differential equations," Computers \& Mathematics with Applications, vol. 52, no. 3-4, pp. 459-470, 2006.

[3] E. A. Rawashdeh, "Numerical solution of fractional integrodifferential equations by collocation method," Applied Mathematics and Computation, vol. 176, no. 1, pp. 1-6, 2006.

[4] A. Secer, M. A. Akinlar, and A. Cevikel, "Efficient solutions of systems of fractional PDEs by the differential transform method," Advances in Difference Equations, vol. 2012, article 188, 2012.
[5] M. Kurulay, A. Secer, and M. A. Akinlar, "A new approximate analytical solution of Kuramoto-Sivashinsky equation using homotopy analysis method," Applied Mathematics \& Information Sciences, vol. 7, no. 1, pp. 267-271, 2013.

[6] Q. Wang, "Homotopy perturbation method for fractional KdVBurgers equation," Chaos, Solitons \& Fractals, vol. 35, no. 5, pp. 843-850, 2008.

[7] M. Li and W. Zhao, "Essay on fractional Riemann-Liouville integral operator versus Mikusinski's," Mathematical Problems in Engineering, vol. 2013, Article ID 635412, 3 pages, 2013.

[8] M. Kurulay, B. A. Ibrahimoǧlu, and M. Bayram, "Solving a system of nonlinear fractional partial differential equations using three dimensional differential transform method," International Journal of Physical Sciences, vol. 5, no. 6, pp. 906-912, 2010.

[9] I. Podlubny, Fractional Differential Equations: An Introduction to Fractional Derivatives, Fractional Differential Equations, to Methods of Their Solution and Some of Their Applications, vol. 198 of Mathematics in Science and Engineering, Academic Press, San Diego, Calif, USA, 1999. 


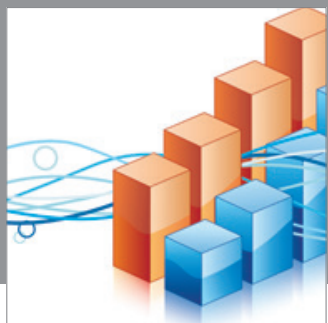

Advances in

Operations Research

mansans

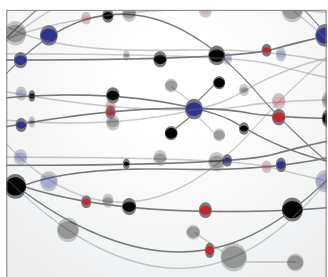

The Scientific World Journal
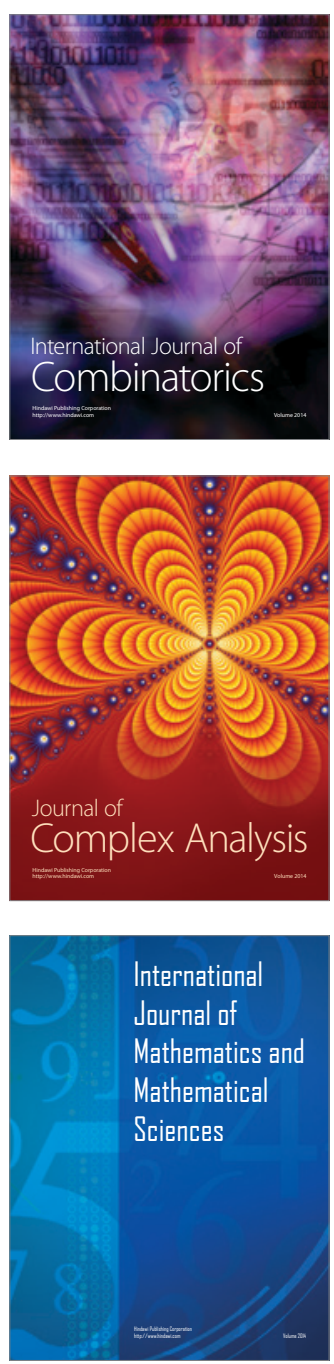
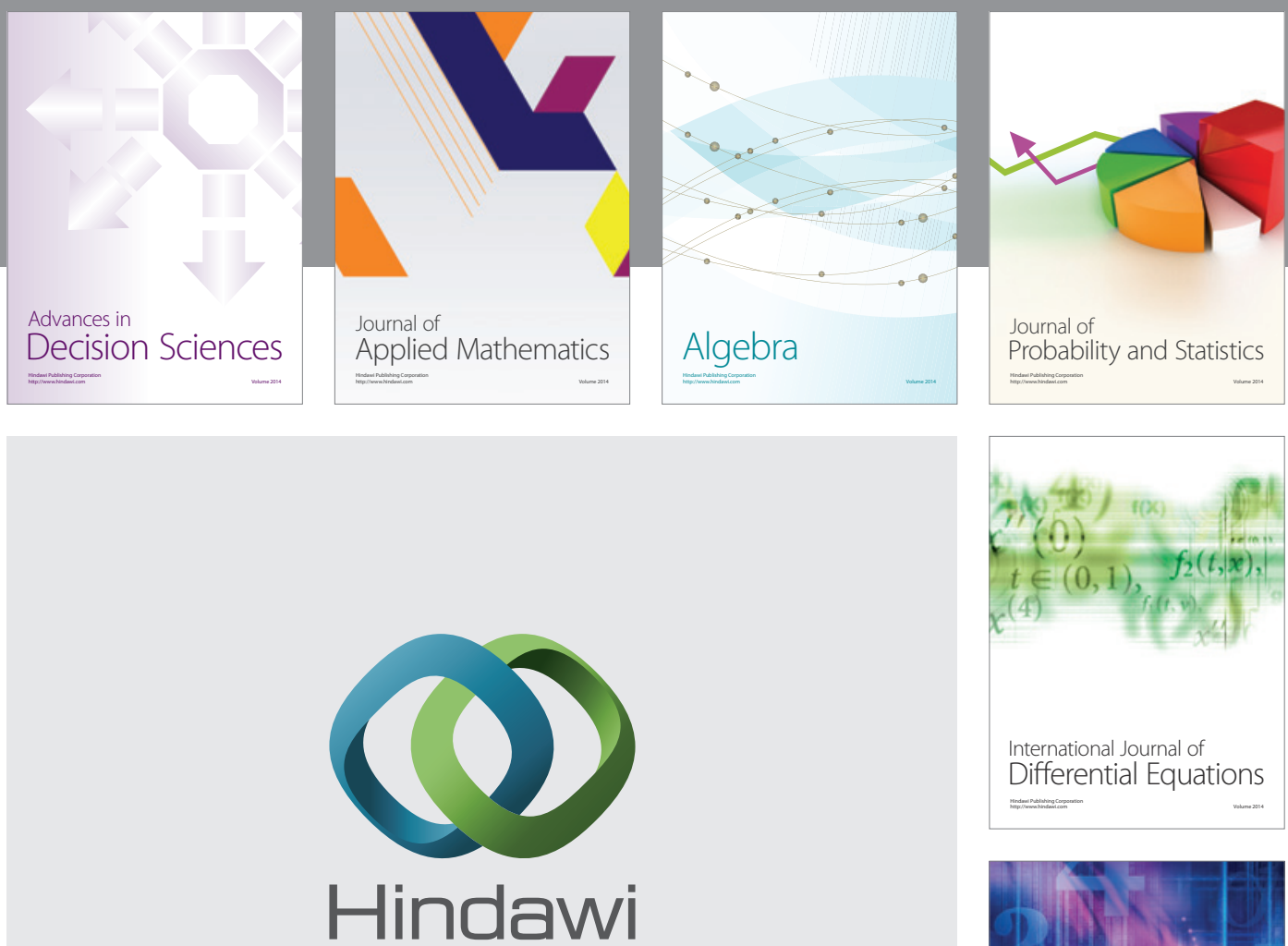

Submit your manuscripts at http://www.hindawi.com
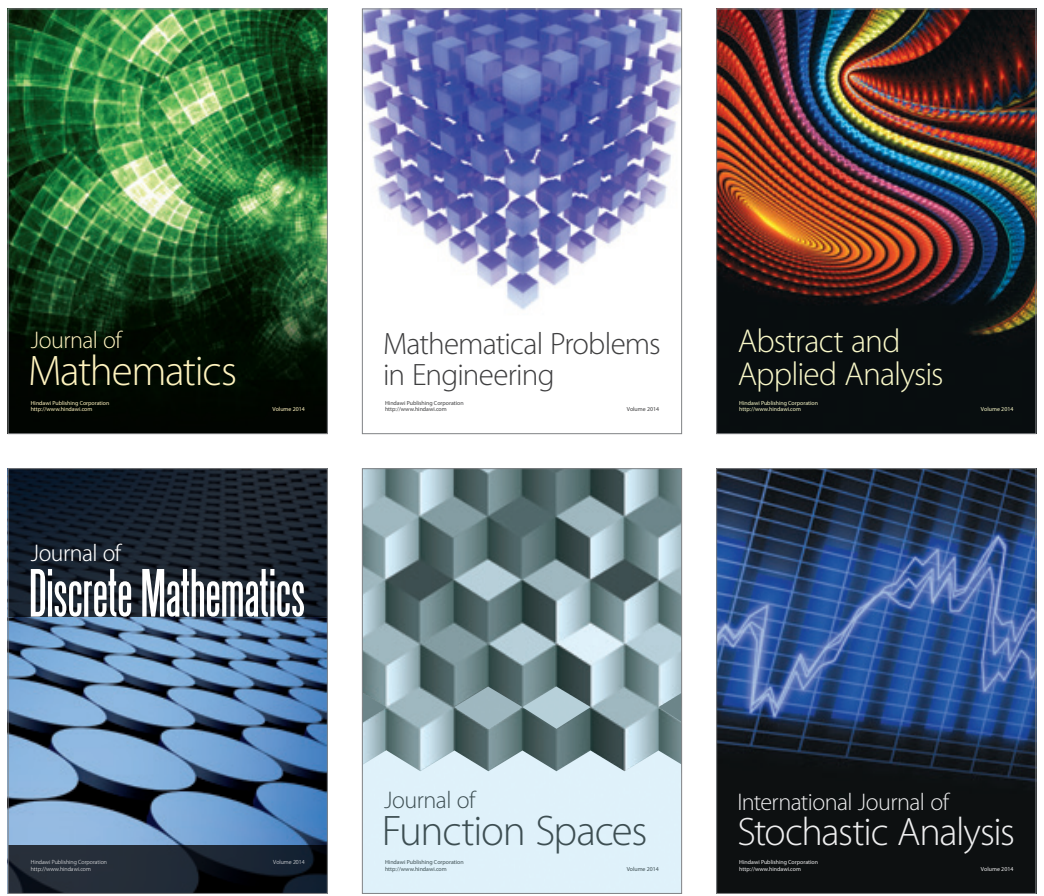

Journal of

Function Spaces

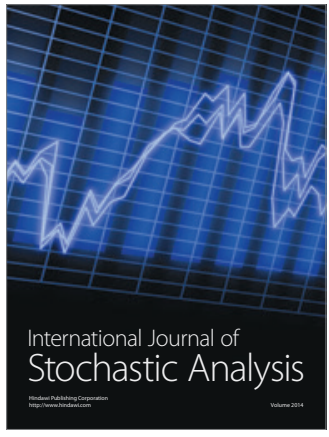

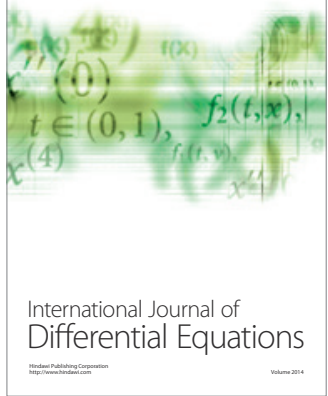
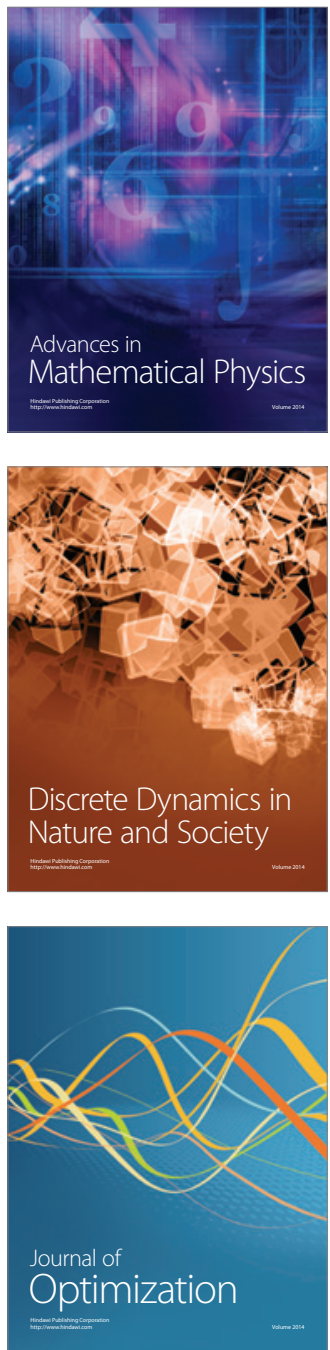